\title{
Targeting FOXA1-mediated repression of TGF- $\beta$ signaling suppresses castration-resistant prostate cancer progression
}

\author{
Bing Song, ${ }^{1}$ Su-Hong Park, ${ }^{1}$ Jonathan C. Zhao, ${ }^{1}$ Ka-wing Fong, ${ }^{1}$ Shangze Li, ${ }^{1}$ Yongik Lee, ${ }^{1}$ Yeqing A. Yang, ${ }^{1}$ Subhasree Sridhar, \\ Xiaodong Lu, ${ }^{1}$ Sarki A. Abdulkadir, ${ }^{2}$ Robert L. Vessella, ${ }^{3}$ Colm Morrissey, ${ }^{3}$ Timothy M. Kuzel, ${ }^{1}$ William Catalona, ${ }^{2}$ \\ Ximing Yang, ${ }^{2}$ and Jindan $\mathbf{Y u}^{1,2,4}$
}

'Division of Hematology/Oncology, Department of Medicine, and Robert H. Lurie Comprehensive Cancer Center, Northwestern University Feinberg School of Medicine, Chicago, Illinois, USA. ${ }^{3}$ Department of Urology, University of Washington, Seattle, Washington, USA. D. Department of Biochemistry and Molecular Genetics, Northwestern University Feinberg School of Medicine, Chicago, Illinois, USA

Prostate cancer (PC) progressed to castration resistance (CRPC) is a fatal disease. CRPC tumors develop resistance to new-generation antiandrogen enzalutamide through lineage plasticity, characterized by epithelial-mesenchymal transition (EMT) and a basal-like phenotype. FOXA1 is a transcription factor essential for epithelial lineage differentiation. Here, we demonstrate that FOXA1 loss leads to remarkable upregulation of transforming growth factor beta 3 (TCFB3), which encodes a ligand of the TCF- $\beta$ pathway. Mechanistically, this is due to genomic occupancy of FOXA1 on an upstream enhancer of the TCFB3 gene to directly inhibit its transcription. Functionally, FOXA1 downregulation induces TCF- $\beta$ signaling, EMT, and cell motility, which is effectively blocked by the TCF- $\beta$ receptor I inhibitor galunisertib (LY2157299). Tissue microarray analysis confirmed reduced levels of FOXA1 protein and a concordant increase in TCF- $\beta$ signaling, indicated by SMAD2 phosphorylation, in CRPC as compared with primary tumors. Importantly, combinatorial LY2157299 treatment sensitized PC cells to enzalutamide, leading to synergistic effects in inhibiting cell invasion in vitro and xenograft CRPC tumor growth and metastasis in vivo. Therefore, our study establishes FOXA1 as an important regulator of lineage plasticity mediated in part by TGF- $\beta$ signaling, and supports a novel therapeutic strategy to control lineage switching and potentially extend clinical response to antiandrogen therapies.

\section{Introduction}

Prostate cancer (PC) is the third leading cause of cancer-related death among men in the United States, where 1 in 8 men are expected to be diagnosed with PC during their lifetime (1). The 5 -year survival rate of localized PC is nearly $100 \%$, but metastatic PC remains a deadly disease. While most advanced PCs initially respond to androgen deprivation therapy (ADT) with tumor regression, a majority of them eventually progress to a resistant disease referred to as castration-resistant prostate cancer (CRPC). Recent use of new-generation antiandrogens such as abiraterone and enzalutamide (Enz) further prolonged patient lives for several months to a year $(2,3)$, yet resistance inevitably develops, ultimately leading to death. Groundbreaking studies have recently shown that CRPC tumors develop resistance by a phenotypic shift from luminal epithelial cells to basal-like cells, facilitated by lineage plasticity $(4,5)$. Thus, there is an urgent need to control this lineage shift and to develop novel therapeutic approaches that can extend clinical response to antiandrogen therapy.

Conflict of interest: The authors have declared that no conflict of interest exists. License: Copyright 2019, American Society for Clinical Investigation.

Submitted: May 21, 2018; Accepted: November 6, 2018.

Reference information: / Clin Invest. 2019;129(2):569-582.

https://doi.org/10.1172/JCl122367.
A diverse array of molecular determinants have been reported in the past years, accounting for resistance to ADT, and out of which the most critical is aberrant activation of androgen receptor (AR) through various mechanisms, including alterations in cofactor activity $(6,7)$. Recent analyses of mutational landscape in prostate tumors have identified FOXA1 as one of the most frequently mutated genes $(8,9)$. In addition, we and others have reported that FOXA1 is downregulated in CRPC as compared with primary PC, suggesting a tumor suppressor role (10-12). We demonstrated the importance of a delicate balance between nuclear FOXA1 and AR protein levels for their cooperated activation in mediating prostate-specific AR transcriptional program (12). In the milieu of low androgen, FOXA1 acts as a key suppressor of residual AR signaling and FOXA1 loss leads to aberrant AR activation and CRPC progression. In addition, we observed cellular transformation of FOXA1-knockdown cells that are representative of epithelial-mesenchymal transition (EMT) and cell dedifferentiation to neuroendocrine phenotype $(13,14)$. Mechanistically, this is caused at least in part by the induction of IL-8 and SLUG, both of which are known targets of transforming growth factor $\beta$ (TGF- $\beta$ ) signaling.

The TGF- $\beta$ pathway has 3 ligands (TGF- $\beta 1,-\beta 2,-\beta 3$ ) that bind to cell surface kinase receptor TGF- $\beta$ receptor 2 (TGFBR2) (15). Once activated, TGFBR2 recruits and phosphorylates TGF- $\beta$ receptor 1 (TGFBR1). Activation of TGFBR1 leads to phosphorylation of the cytoplasmic R-SMAD transcription factors (SMAD2/3) 
A

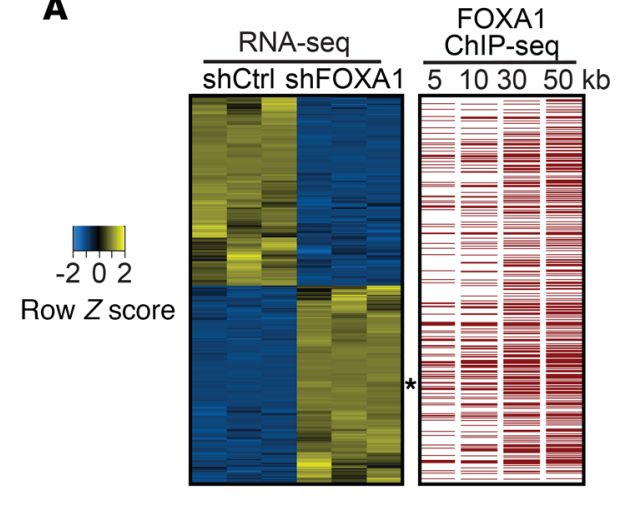

B

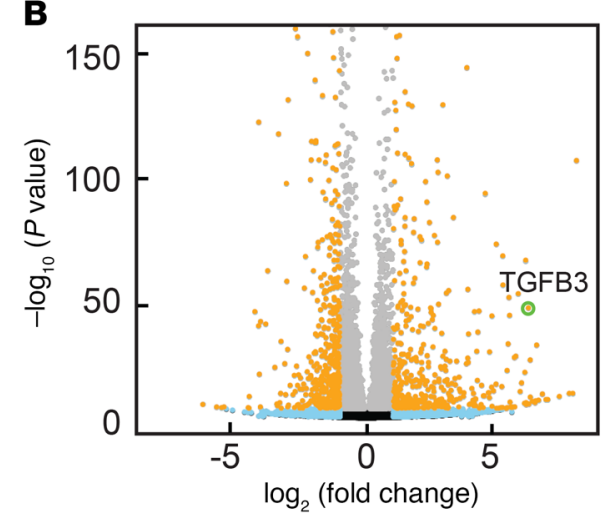

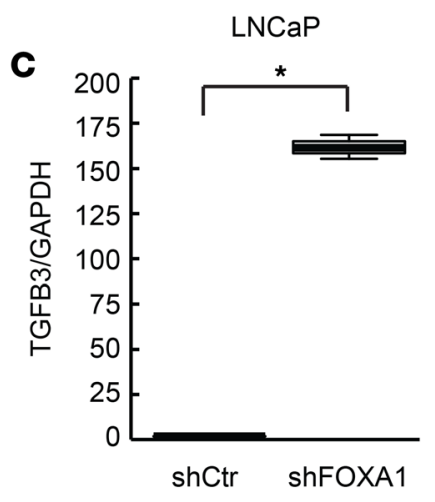

D

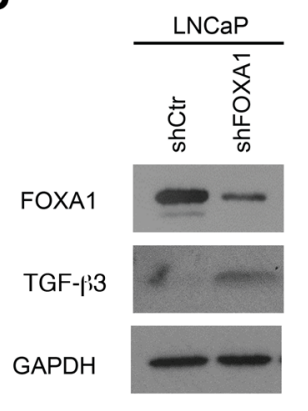

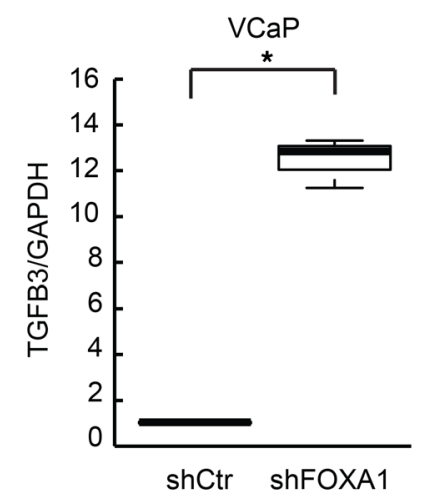

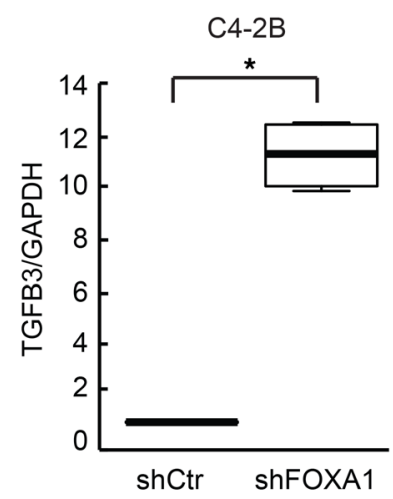

$\mathbf{E}$
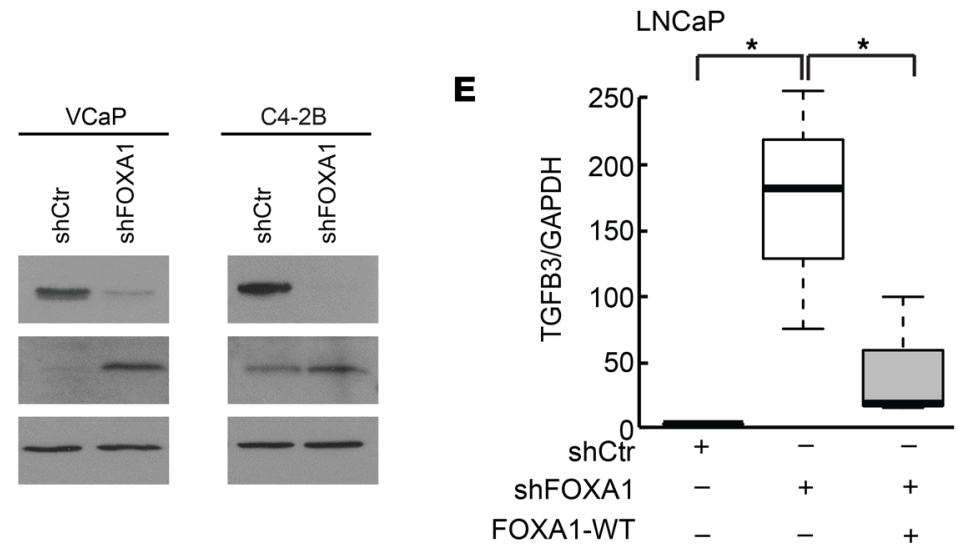

Figure 1. FOXA1 suppresses TCFB3 gene transcription. (A) Heat map of differentially expressed genes in LNCaP cells infected with control (shCtr) versus shFOXA1 profiled by RNA-seq. FOXA1-regulated genes were selected by DESeq2 with at least 2-fold changes in expression (RPKM) and Benjamini-Hochberg adjusted $P$ values less than 0.01 . Each row corresponds to one gene and each column one sample. Data shown are $\log _{2}$ RPKM values. The 4 bar plots on the right indicate FOXA1 ChIP-seq binding within $5 \mathrm{~kb}, 10 \mathrm{~kb}, 30 \mathrm{~kb}$ or $50 \mathrm{~kb}$ of transcription start site (TSS). (B) Volcano plot showing differentially expressed genes between shCtrl and shFOXA1 LNCaP cells. The $x$ axis represents $\log _{2}$ (shFOXA1/shCtrl) for each gene, and the $y$ axis shows statistical significance. Orange dots indicate differentially expressed genes (adjusted $P \leq 0.001$ ); light blue dots are genes with insignificant changes; gray dots are genes with less than 2-fold changes. TCFB3 gene is highlighted by a green circle. (C and D) TCFB3 gene expressions (C) and TCF- $\beta 3$ protein levels (D) are upregulated upon FOXA1 knockdown. LNCaP, VCaP and C4-2B cells were infected with shCtr or shFOXA1 lentivirus followed by puromycin selection, and then analyzed by qRT-PCR and Western blots $\left(n=3\right.$, $\left.{ }^{*} P<0.05\right)$. (E) FOXA1-WT overexpression rescues FOXA1 loss induced TCFB3 gene expression. LNCaP cells were infected with either shCtr or shFOXA1-knockdown lentivirus with or without FOXA1-WT reexpressing lentivirus and harvested for qRT-PCR analysis $\left(n=3,{ }^{*} P<0.05\right)$.

and subsequent heteromeric formation with the co-SMAD (SMAD4). The SMAD2/3-SMAD4 complex then translocates to the nucleus to regulate the expression of genes controlling diverse cellular processes, including apoptosis, cell proliferation, EMT, cell invasion, and immune regulation in a context-dependent fashion (16). In advanced cancer, TGF- $\beta$ signaling was strongly linked to increased cell invasiveness and tumor metastasis (17). In
PC, TGF- $\beta$-induced expression of vimentin is associated with biochemical recurrence (18) and TGF- $\beta$ from bone marrow-derived mesenchymal stem cells promotes metastasis of PC (19). In particular, upregulation of TGF- $\beta 3$ has been shown to have strong effects on the migratory and invasive behaviors of PC cells (20). Such results have fostered strong interests in therapeutic targeting of this pathway in advanced cancer, and anti-TGF- $\beta$ therapies are 
under active development. LY2157299 monohydrate (also termed galunisertib), an inhibitor of TGF- $\beta$ receptor I kinase, has been characterized in various preclinical studies. It was shown to inhibit SMAD phosphorylation (pSMAD), reverse EMT, and reduce tumor cell motility (21), and may be effective for hepatocellular carcinoma. First-in-human dose studies of LY2157299 have reported cardiac safety of this drug in humans and demonstrated some antitumor activity in patients with glioma $(22,23)$.

In the present study, we report a role of FOXA1 as a transcriptional repressor. FOXA1 loss drastically increased the expression of TGFB3 and its receptors, inducing TGF- $\beta$ signaling, EMT, and cell motility, which can be blocked by LY2157299. We confirmed the loss of FOXA1 and gain of TGF- $\beta$ signaling in human CRPC tissues as compared with primary PC, and demonstrated a synergy between Enz and LY2157299 in inhibiting PC cell growth and invasion in vitro and suppressing CRPC tumor growth and metastasis in vivo.

\section{Results}

Integrative genomics analyses reveal TGFB3 as a target of FOXA1-mediated transcriptional repression. FOXA1 is a pioneer factor that recruits $\mathrm{AR}$ to accessible chromatin and thus mediates its transcriptional activities $(12,24)$. We and others have shown that AR can act as a transcription repressor on some target genes $(25,26)$. As we have recently found that FOXA1 can also directly inhibit gene expression $(13,14)$, we asked whether and how FOXA1 might also act as a transcriptional repressor. We first performed RNA-Seq profiling of LNCaP cells with control and FOXA1 knockdown. Bioinformatics analysis of triplicate experiments revealed slightly more genes that are induced (591 genes) by FOXA1 than repressed ( 565 genes) (Figure 1A). Integrative analysis with FOXA1 ChIP-seq data showed that approximately $32 \%$ of FOXA1-induced genes and 21\% of FOXA1-repressed genes contained at least 1 FOXA1 binding event within $5 \mathrm{~kb}$ of their promoters, suggesting that FOXA1 acts as a transcriptional repressor on a significant number of genes, albeit on fewer genes than the induced ones. As FOXA1 is known to bind enhancers, we expanded the analysis to enhancer elements and observed up to $71 \%$ of FOXA1-induced and $58 \%$ of FOXA1-repressed genes that contained at least 1 FOXA1 binding event within $50 \mathrm{~kb}$ around their promoters (Figure 1A). Ranked among the top FOXA1-repressed genes is TGFB3, which contained a strong FOXA1 binding event within $5 \mathrm{~kb}$ of its promoter and is upregulated by more than 60-fold upon FOXA1 depletion (Figure 1B).

To verify that TGFB3 is repressed by FOXA1, we performed FOXA1 knockdown in multiple PC cell lines (Supplemental Figure 1A; supplemental material available online with this article; https://doi.org/10.1172/JCI122367DS1). Quantitative RT-PCR (qRT-PCR) analysis using gene-specific primers (Supplemental Table 1) showed that TGFB3 expression is upregulated upon FOXA1 depletion by approximately 140-, 14-, and 10-fold in LNCaP, VCaP, and C4-2B cells, respectively (Figure 1C). Similar results were observed using another shRNA targeting 3'UTR of the FOXA1 gene (Supplemental Figure 1, B and C). Consistent with this increase in its mRNA levels, TGF- $\beta 3$ protein was also strongly increased, as demonstrated by Western blotting in all 3 cell lines tested (Figure 1D). Moreover, reintroduction of ectopic FOXA1 to these cells partially dampened the induction of TGFB3, confirming the specificity of this regulation (Figure $1 \mathrm{E}$ and Supplemental Figure 1D). In aggregate, our results support that FOXA1 may act as a transcriptional repressor and suggest TGFB3 as a top target.

FOXA1 binds to the TGFB3 enhancer to inhibit its expression. Our previous work has shown that FOXA1 directly represses IL-8 and SLUG gene expression in PC $(13,14)$. We next investigated the possibility that TGFB3 is also a direct target of FOXA1-mediated transcriptional repression. Analysis of FOXA1 ChIP-seq previously performed in LNCaP cells (13) identified strong FOXA1 binding at an enhancer of approximately $3.7 \mathrm{~kb}$ upstream of the TGFB3 gene promoter (Figure 2A). Further, FOXA1 binding at this enhancer was decreased by $27 \%$ upon FOXA1 knockdown, supporting an authentic FOXA1 binding event. Similarly, FOXA1 occupied the same site in VCaP cells (Supplemental Figure 2A). To further validate these observations, we carried out FOXA1 ChIP-qPCR analysis. Our data demonstrated very strong FOXA1 occupancy at the TGFB3 enhancer, whereas the TGFB3 promoter was marked with a relatively weaker but still highly significant FOXA1 enrichment (Figure 2B and Supplemental Figure 2B). Further, FOXA1 occupancy on both sites was greatly reduced in FOXA1-knockdown cells, whereas FOXA1 was not significantly enriched at negative control sites, KIAA0066, and an intermediate region between TGFB3 promoter and enhancer. In addition, ChIP-qPCR showed significantly enriched occupancy by active RNA polymerase II (PolII p-Ser-5) and active histone mark H3K4me3 at the TGFB3 promoter following FOXA1 knockdown, being concordant with the increased transcription of TGFB3 (Figure 2, C and D).

To demonstrate that FOXA1 binding at the upstream enhancer indeed inhibits TGFB3 gene transcription in vivo, we used a lentiviral CRISPR/Cas9 editing system to delete the enhancer elements with small guide RNAs (sgRNAs). Motif analysis revealed 3 consensus forkhead (FKHD) motifs localized within the ChIP-seq FOXA1 binding peak, and sgRNAs were designed to delete a fragment covering all 3 motifs (Figure $2 \mathrm{E}$ ). As LNCaP is a polyploidy cell line, it is challenging to delete a target region from all copies of chromosomes using CRISPR/Cas9. Further, as LNCaP cells lose their normal morphology when grown in isolation, it is not practical to select an individual clone to grow out using CRISPR/ Cas9 deletion. We thus opted to analyze a heterogeneous population of edited and unedited cells. PCR analysis of genomic DNA from a pooled population revealed approximately $25 \%$ of editing, comparing the PCR products of the WT and deleted alleles (Figure 2F). Importantly, qRT-PCR analysis demonstrated that CRISPR-mediated enhancer deletion in only one-fourth of the cell population was able to substantially restore the transcription of TGFB3 gene, comparing cells transfected with TGFB3-targeting gRNAs to those with control gRNAs. TGFB3 expression in the pooled population was further increased upon FOXA1 knockdown (Figure 2G and Supplemental Figure 2C), which can be accounted for by the unedited cells. Taken together, these results strongly support that FOXA1 binds to an upstream enhancer to directly repress TGFB3 transcription.

FOXA1 loss activates TGF- $\beta$ signaling in prostate cancer cells. As FOXA1 depletion substantially increased the expression of TGFB3, we next asked whether it might lead to activation of TGF- $\beta$ signal transduction. This may establish TGF- $\beta$ as a therapeutic target, as previous studies have reported FOXA1 downregulation in 
A

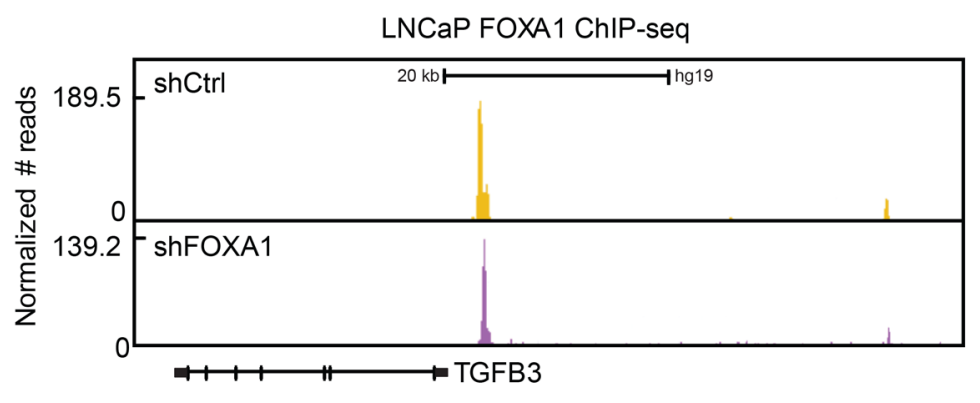

C RNA Polll p-Ser5 ChIP

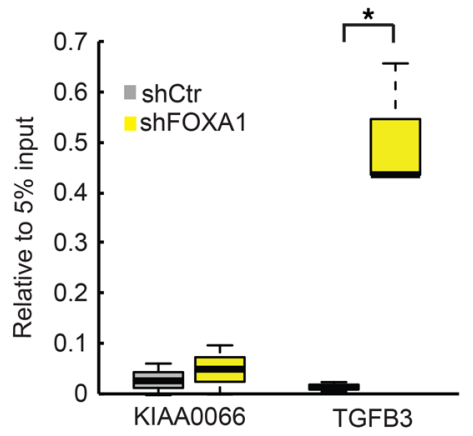

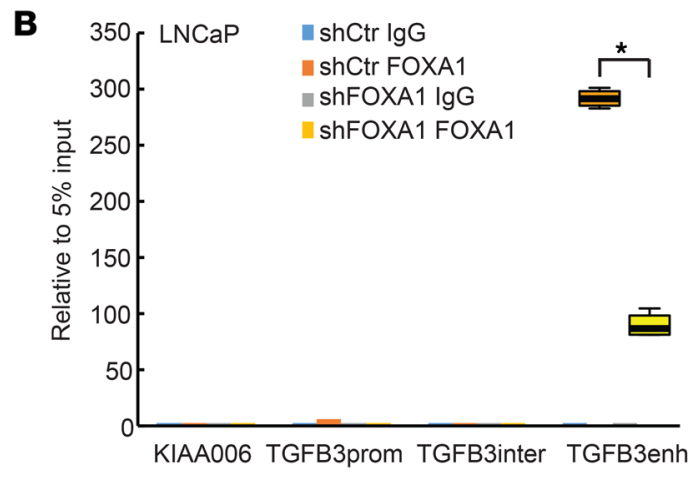

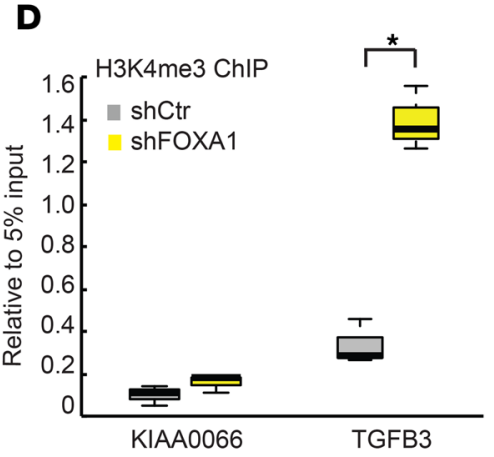

E

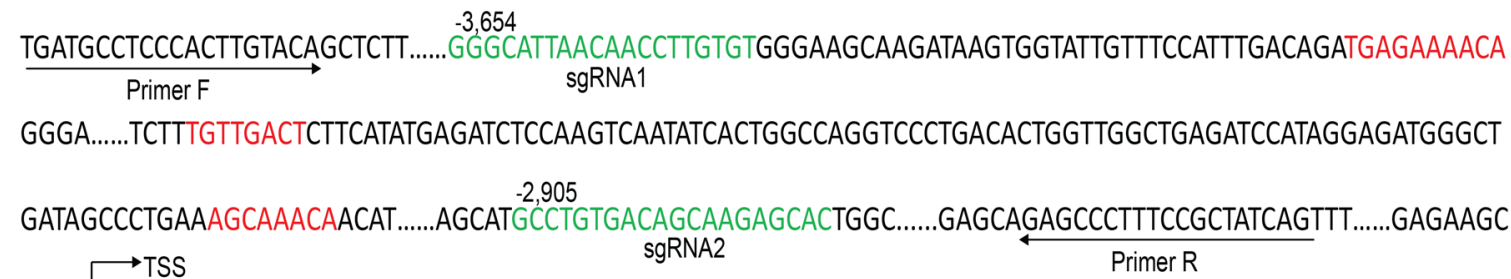

AGGGGACAGAAGCAATGGCCGAGGC
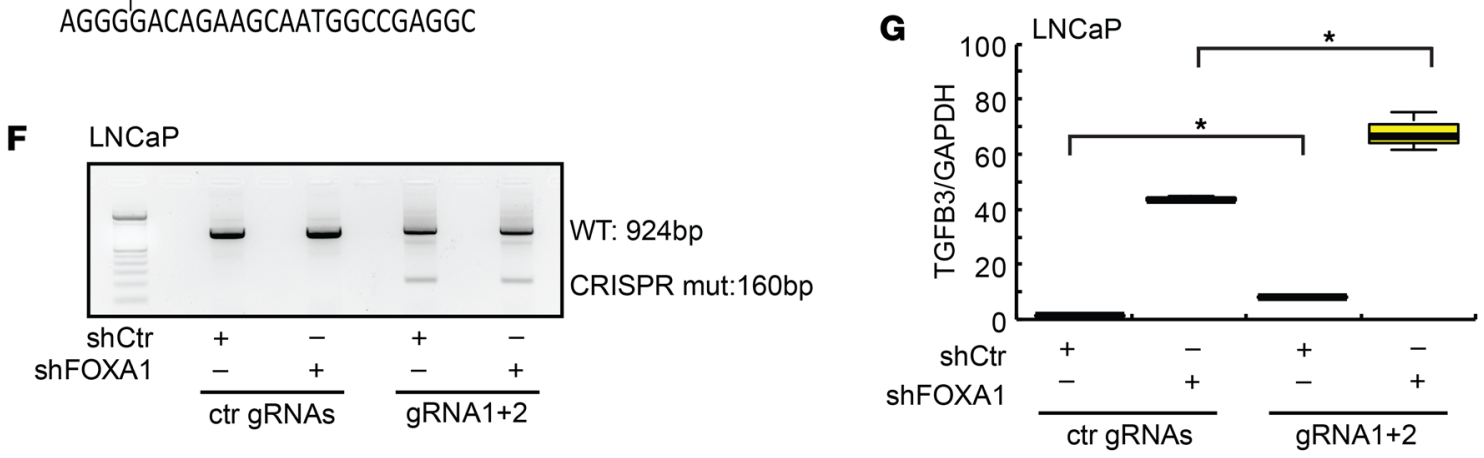

Figure 2. FOXA1 protein binds to an upstream enhancer of TGFB3 to inhibit its transcription. (A) Genome browser view showing FOXA1 binding at an upstream TGFB3 enhancer. FOXA1 ChIP-seq was performed in LNCaP shCtr and shFOXA1 cells as previously described (12). The magnitudes of FOXA1 binding peak at this enhancer in shCtr and shFOXA1 were 189.5 and 139.2, respectively. (B) FOXA1 and IgG ChIP were performed in shCtrl and shFOXA1 LNCaP cells and subjected to qPCR using primers flanking the promoter (prom), enhancer (enh), and an intermediate (inter) region (as a negative control) of the TCFB3 gene. KIAA0066 was used as a negative control $\left(n=3,{ }^{*} P<0.05\right)$. (C and D) ChIP of RNA Pol II (phosphorylated at Ser5) (C) and H3K4me3 (D) was performed in LNCaP cells with shCtr or shFOXA1 knockdown and subjected to qPCR analysis with primers for TCFB3 enhancer and KIAA0066 control gene. (E) Schema of CRISPR/Cas9 editing of FOXA1-bound TCFB3 enhancer region. Distance to TSS of the TGFB3 gene is labeled. The sgRNA sequences are shown in green and FKHD motifs in red. (F and G) LNCaP cells were infected with CRISPR/Cas9 along with control (ctr) or TCFB3 enhancer-targeting sgRNA1 and 2 (gRNA1+2), which were then subjected to control (shCtr) or FOXA1 (shFOXA1) knockdown. Total RNA and genomic DNA were isolated from a pooled population of cells under each condition. Genomic DNA were subjected to PCR using primers $F$ and $R$ as labeled in panel E. The top PCR band indicates the full-length WT PCR amplicons, whereas the lower band indicates the CRISPR-edited PCR amplicon, with about $25 \%$ editing rate (F). RNA were subjected to qRT-PCR to measure TCFB3 gene expression, and normalized to GAPDH (C). 
CRPC and neuroendocrine PC (NEPC) patient tumors $(10,13,14)$. We first attempted to identify a relevant PC model for the study of TGF- $\beta$ signaling by examining the expression of essential TGF- $\beta$ receptors, TGFBR1 and TGFBR2, in a panel of PC cell lines. Our data revealed that TGFBR1 was expressed at abundant levels in most of the PC cell lines, whereas TGFBR2 expression was much more variable with magnitudes of differences between cell lines (Supplemental Figure 3, A and B). This is consistent with previous reports of TGFBR2 repression in some PCs (27). TGFBR2 was barely expressed in most of the AR-positive cell lines, with VCaP having the highest expression. In order to generate highly sensitive AR-positive PC models, we overexpressed the TGFBR2 gene in LNCaP and C4-2B cells to generate TGFBR2 (RII) stable cell lines. Next, we treated these cell lines with TGF- $\beta 1$ to test their responsiveness to TGF- $\beta$ ligands. In concordance with their respective TGFBR2 levels, both LNCaP-RII and C4-2B-RII stable cells were highly responsive to TGF- $\beta 1$ stimulation, as indicated by a dramatic increase of pSMAD2 levels, whereas the control LNCaP and C4-2B cells with empty vector expression failed to respond (Figure 3A and Supplemental Figure 3C). On the other hand, TGFBR2-positive VCaP cells showed a weak but consistent increase of pSMAD2 upon TGF- $\beta 1$ stimulation (Figure 3A, right panel). In all responsive cell lines, TGFBR1 inhibitors (LY2157299 or SB525334) are highly effective in blocking TGF- $\beta$ signaling.

Next, we attempted to investigate how FOXA1 regulates TGF- $\beta$ signaling. As TGF- $\beta 3$ is a secreted protein, we tested whether conditioned medium (CM) from FOXA1-knockdown cells was able to induce TGF- $\beta$ signaling in parental cells. First, we treated the highly responsive LNCaP-RII cells with CM collected from LNCaP-RII cells with either control or FOXA1 knockdown. Western blot analysis showed a clearly increased amount of pSMAD2 in cells treated with FOXA1-knockdown CM (Figure 3B). As activated pSMAD2 is known to translocate into nucleus where it binds Smad binding elements (SBEs) to regulate target gene transcription (28), we transfected a SBE-driven luciferase reporter construct into LNCaP-RII cells. Luciferase assay demonstrated that CM from FOXA1-knockdown cells greatly increased SBE-driven luciferase reporter activities, in concordance with increased pSMAD levels. To further explore the use of an endogenous system, we stimulated VCaP cells with CM derived from either control or FOXA1-depleted VCaP cells. Western blotting and luciferase assays confirmed similar activation of TGF- $\beta$ signaling, as indicated by pSMAD2 level and SBE luciferase activities, by $\mathrm{CM}$ from FOXA1-depleted cells (Figure 3C).

Once we confirmed that CM from FOXA1-depleted cells is sufficient to turn on TGF- $\beta$ signaling, we asked whether the TGF- $\beta$ pathway may be activated in FOXA1-depleted LNCaP cells. Interestingly, gene set enrichment analysis (GSEA) showed a trend of significant upregulation of KEGG_TGFB_pathway genes in LNCaP cells following FOXA1 knockdown (Supplemental Figure 3 , D and E). Almost all TGF- $\beta$ ligands and receptors were upregulated, except TGFBR1, which was reduced by 1.6-fold but was still highly abundant with a RPKM (reads per kilobase of transcript, per million mapped) of about 2.8 (Figure 3D). In particular, we observed that TGFBR2, which is dynamically regulated during cancer progression (27), was increased upon FOXA1 knockdown by 2.9 -fold, from a RPKM of 0.14 to 0.40 . This dramatic increase of TGFBR2 may sensitize LNCaP cells to TGF- $\beta$ ligands, accounting at least partially for the overall activation of TGF- $\beta$ signaling in FOXA1-knockdown LNCaP cells. Through qRT-PCR analysis, we confirmed that TGFBR2 expression increased upon FOXA1 depletion by approximately 5.8-, 1.6-, and 6-fold in LNCaP, VCaP, and C4-2B cells, respectively (Figure 3E).

To elucidate global correlation between FOXA1 and TGF- $\beta$ signaling, we first performed expression profiling of LNCaP-RII cells stimulated with either control or TGF- $\beta 3$ and derived TGF- $\beta 3-$ induced and -repressed gene signatures. GSEA analysis revealed that TGF- $\beta 3$-induced genes were significantly upregulated in FOXA1-knockdown LNCaP cells relative to control cells, whereas TGF- $\beta 3$-repressed genes were remarkably downregulated (Figure $3 \mathrm{~F}$ and Supplemental Figure 3F). Most importantly, treatment of FOXA1-knockdown cells with LY2157299 decreased TGF- $\beta 3-$ induced gene expression, whereas it restored TGF- $\beta 3$-repressed gene expression, fully rescuing the effects of FOXA1 loss (Figure $3 G$ and Supplemental Figure 3G). Further, analysis of SU2C and TCGA PC data sets revealed that TGF- $\beta 3$-induced genes were enriched for upregulation, whereas TGF- $\beta 3$-repressed genes were decreased in FOXA1-low PC (Supplemental Figure 3, H and I), supporting the pathological relevance of the FOXA1-TGFB3 axis. Examination of limited cases with FOXA1 mutations did not reveal a strong correlation with TGF- $\beta$ signature, potentially due to their variable levels of FOXA1 expression. Altogether, we conclude that FOXA1 loss increased the expression of multiple TGF- $\beta$ ligands and receptors, leading to active TGF- $\beta$ signaling in PC.

FOXA1 loss induced cell invasion and EMT, which is blocked by TGF- $\beta$ receptor I inhibitors. Studies have previously reported that FOXA1 loss induces EMT and increases PC cell invasion $(13,14)$. To determine whether its target gene TGFB3 produces similar effects, we treated LNCaP-RII, C4-2B-RII, and VCaP cells with recombinant TGF- $\beta 3$ ligand. Our results confirmed enhanced cell invasion in all 3 cell lines following TGF- $\beta 3$ stimulation, which was blocked by LY2157299 or SB525334 treatment (Figure 4A and Supplemental Figure 4, A and B). Next, we investigated the functional significance of FOXA1 regulation of TGFB3 expression. Invasion assays using Matrigel revealed that FOXA1 loss indeed induced LNCaP cell invasion, which was blocked by concurrent LY2157299 treatment (Figure 4B). Similar results were also observed in VCaP and C4-2B cells (Figure 4C and Supplemental Figure $4 \mathrm{C}$ ), indicating TGF- $\beta$ signaling as an important downstream mediator of FOXA1 loss-induced cell invasion. As controls, we showed that LY2157299 did not affect cell proliferation, thus precluding the potential of different cell numbers in confounding cell invasion (Supplemental Figure 4, D and E). In addition, we found that LY2157299 treatment did not affect the invasion of their parental cell lines that do not express a sufficient amount of TGFBR1, supporting target specificity (Supplemental Figure 4, F and G).

Interestingly, we observed that FOXA1 knockdown in LNCaP cells led to a more dispersed and fibroblastic morphology that is typical of EMT, which is consistent with our previous reports (13, 14). Importantly, LY2157299 treatment partially reversed the effects, leading to better-defined, epithelial-like cells (Figure 4D and Supplemental Figure 4H). Further, immunofluorescent staining showed that control LNCaP cells exhibited normal 
A

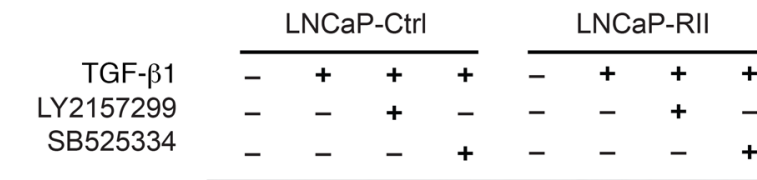

HA(TGFB-RII)

pSmad2

GAPDH
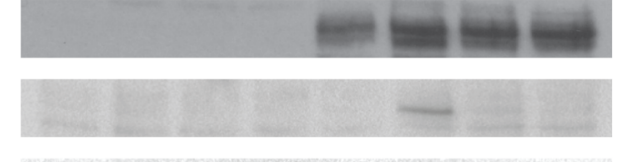

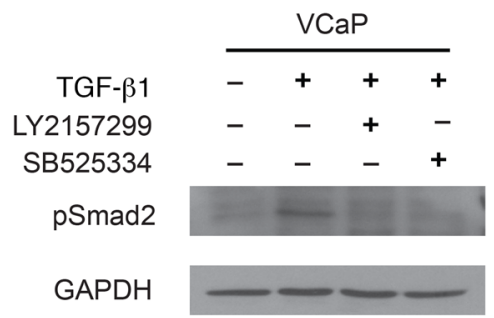

GAPDH
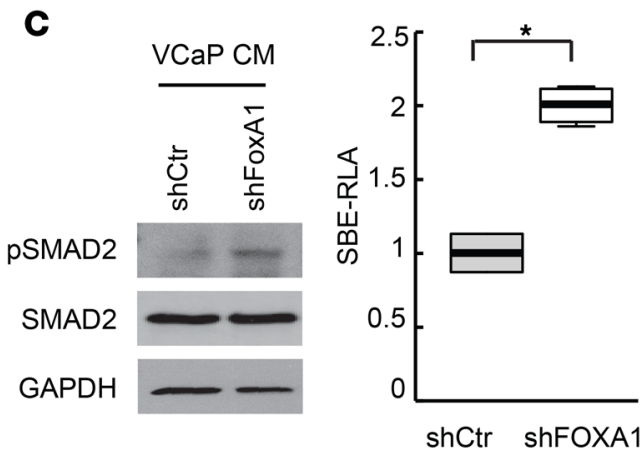

\section{D}

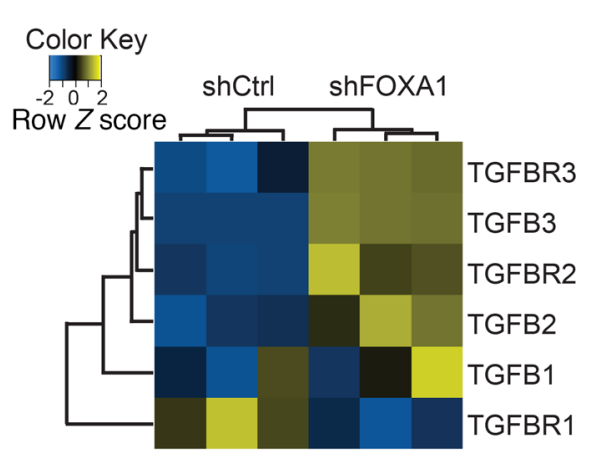

F

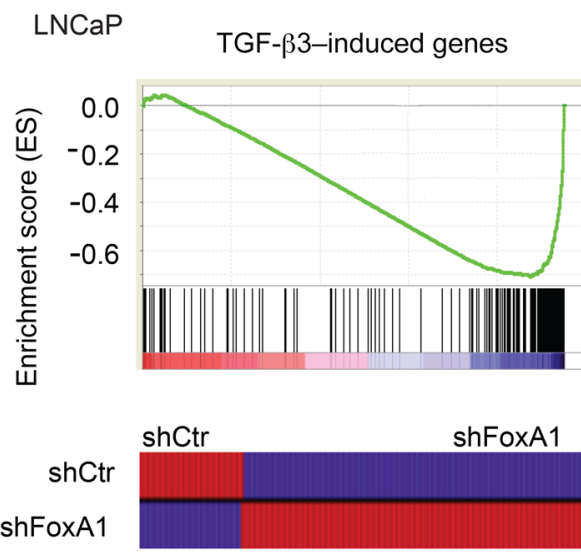

E

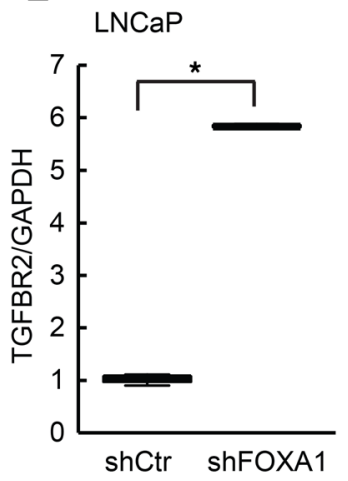

C4-2B

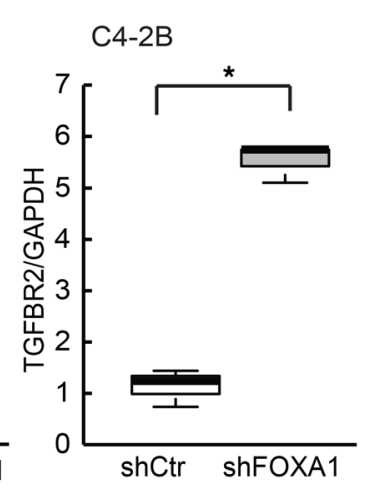

NES $=-2.78$ $P=0.000$ $\mathrm{FDR}=0.000$

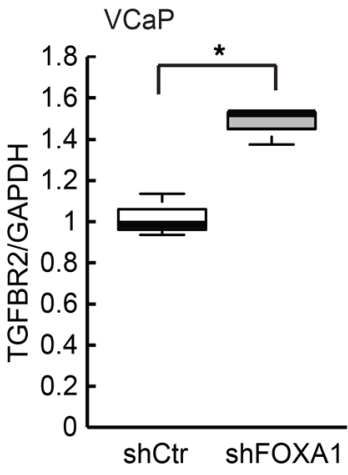

G LNCaP TGF- $\beta 3$-induced genes
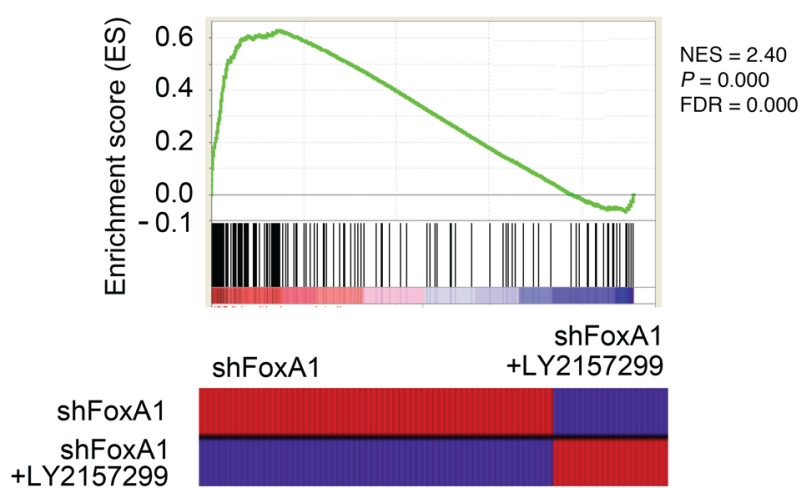
Figure 3. FOXA1 loss activates TGF- $\beta$ signaling in PC cells. (A and B) LNCaP-Ctrl, LNCaP-RII, and VCaP cell lines were treated with DMSO or $5 \mathrm{ng} / \mathrm{ml}$ TGF- $\beta 1$ ligand for 4 days, with or without subsequent $10 \mu \mathrm{M}$ LY2157299 treatment for 1 day. Cells were then collected and analyzed by Western blotting. (B and C) Conditioned medium (CM) from FOXA1-knockdown cells induces TCF- $\beta$ signaling. Conditioned media were collected from stable shCtr and shFOXA1 LNCaP-RII (B) or VCaP (C) cells and added to their corresponding parental cell line for 3 days for Western blot analysis (left panels). Conditioned media were also added to LNCaP-RII or VCaP cells, which had been transfected with a SBE-luciferase reporter construct for 2 days and then harvested for luciferase assay (right panels). RLA: relative luciferase activity; $n=3$; ${ }^{*} P<0.05$. (D) Heatmap showing the expression TCF- $\beta$ pathway genes in control and FOXA1-knockdown LNCaP cells as profiled by RNA-seq. (E) TGFBR2 gene expressions are upregulated upon FOXA1 knockdown. LNCaP, VCaP, and C4-2B cells were infected with shCtr or shFOXA1-knockdown lentivirus followed by puromycin selection, and then analyzed by qRT-PCR $\left(n=3,{ }^{*} P<0.05\right)$. ( $F$ and $\left.\mathbf{G}\right)$ GSEA and heatmaps showing the enriched expression of a TCF- $\beta 3$-induced gene signature in FOXA1-knockdown cells (F), which were rescued by $10 \mu \mathrm{M}$ LY2157299 treatment (C).

E-cadherin staining at the cell membrane on cell-cell contacts, showing sharp fluorescent lining of the cell membrane. However, this well-defined pattern of E-cadherin membrane staining was lost in FOXA1-depleted cells, but was rescued upon concurrent LY2157299 treatment. Taken together, our results demonstrated that enhanced TGF- $\beta$ signaling is a critical mediator of FOXA1loss-induced EMT and cell motility, which can be effectively blocked by LY2157299.

The FOXA1-TGF- $\beta$-SMAD axis is deregulated in metastatic castration-resistant prostate cancer. We and others have previously reported that the FOXA1 mRNA level is decreased in CRPC as compared with primary PC in a majority of publically available PC data sets $(10,13)$. To validate the clinical relevance of the FOXA1TGF- $\beta$-SMAD axis, we first examined by immunohistochemistry the protein levels of FOXA1 in tissue microarrays (TMAs) of human PC tissues. Consistent with its role as an epithelial transcription factor, FOXA1 showed strong and punctuated nuclear staining exclusively in prostate epithelial cells (Figure 5A). Further, more than $90 \%$ of the cases of primary PC stained positive for FOXA1, half of which showed very strong staining (Figure 5B). In great contrast, nearly $60 \%$ of the metastatic CRPC cases exhibited absent FOXA1 staining and another 15\% showed weak staining, although it is also clear that some CRPC tissues maintained moderate to strong FOXA1 staining.

In matched sets of TMAs, we also performed immunohistochemistry for $\mathrm{pSMAD} 2$ as a marker of TGF- $\beta$ signaling. The staining of pSMAD2 is mostly in the nuclei (Figure 5C). In contrast to reduced FOXA1 staining from primary PC to CRPC, we found an increased trend of pSMAD staining upon disease progression, although the difference between primary PC and CRPC was not as striking as FOXA1 staining. About $30 \%$ of primary PC cases were pSMAD2-negative, whereas almost all CRPC cases stained positive for pSMAD2 (Figure 5D). Out of these, nearly $50 \%$ of the CRPC cases exhibited strong staining, whereas only $20 \%$ of primary tumor cases showed strong pSMAD2 staining. Therefore, TMA of clinical specimens confirmed the loss of FOXA1 and the elevation of TGF- $\beta$ signaling in metastatic CRPC as compared with primary $\mathrm{PC}$.
Enzalutamide and LY2157299 showed synergistic effects in inhibiting prostate cancer progression in vitro. As it has been largely impractical in the clinic to restore the expression and function of a lost gene such as FOXA1, we proposed to target its key downstream pathways that are elevated. We have recently shown that FOXA1 loss leads to CRPC progression through upregulation of AR signaling in the milieu of low androgen (12). In the present study, we present evidence that FOXA1 knockdown induces TGF- $\beta$ signaling. Thus, it may be beneficial to use TGF- $\beta$ inhibitors in conjunction with AR antagonists in CRPC. This strategy is appealing also because Enz alone has been previously shown to induce EMT and xenograft tumor metastasis (29), which may be blocked by TGF- $\beta$ pathway inhibition. On the other hand, TGF- $\beta$ signaling has been reported to enhance AR signaling, which should be abolished by concurrent use of Enz. To test this hypothesis, we treated VCaP cells with TGF- $\beta 3$, Enz, LY2157299, or their combinations (Figure $6, \mathrm{~A}$ and B). Western blot analysis confirmed that TGF- $\beta 3$ stimulation indeed increased prostate-specific antigen (PSA) level, in addition to inducing pSMAD. On the other hand, Enz treatment, though it reduced PSA, inadvertently increased pSMAD2. Most importantly, Enz and LY2157299 drug combination reduced both PSA and pSMAD2 levels.

To examine the functional effects of this drug combination, we next treated VCaP with either Enz or LY2157299 as a single agent or in combination. Cell invasion assay revealed that LY2157299 suppressed VCaP cell invasion as expected and also reduced invasion of Enz-treated cells (Figure 6C). To better model CRPC in the tumor environment with increased TGF- $\beta$ signaling, we pretreated VCaP cells with TGF- $\beta 3$ before testing for drug effects. Cell invasion assays showed strong synergistic effects of Enz and LY2157299 in suppressing VCaP cell invasion in the milieu of active TGF- $\beta$ signaling (Figure 6D). As controls, we examined proliferation of these cells. First, we found that either TGF- $\beta 3$ stimulation or LY2157299 as a single agent did not alter VCaP cell growth (Supplemental Figure 5A). Importantly, we observed a synergy between LY2157299 and Enz in inhibiting VCaP cell growth either in the presence or absence of TGF- $\beta 3$ stimulation.

To further validate the drug combination in additional models, we examined LNCaP-RII and its control cells. We found that Enz treatment significantly increased, whereas LY2157299 decreased, LNCaP-RII cell invasion (Figure 6E). Moreover, drug combination showed that LY2157299 blocked Enz-induced invasion of LNCaP-RII cells, consistent with VCaP data. By contrast, Enz treatment did not seem to increase LNCaP cell invasion, potentially due to cell growth inhibition, and drug combination did not strongly affect the invasiveness of $\mathrm{LNCaP}$ cells, which have low endogenous TGF- $\beta$ signaling (Supplemental Figure 5B). Interestingly, unlike $\mathrm{VCaP}$ cells, the drug combination did not appear to inhibit LNCaP control and LNCaP-RII cell growth (Supplemental Figure 5C). In aggregate, our data demonstrated a consistent role of LY2157299 in strongly inhibiting TGF- $\beta$-induced and Enz-induced PC cell invasion.

Enzalutamide and LY2157299 drug combination blocked xenograft prostate tumor growth and metastasis. As we had demonstrated synergistic effects of Enz and LY2157299 in suppressing PC in vitro, we next sought to test whether simultaneous treatment with LY2157299 would sensitize CRPC tumors to Enz in animal 
A
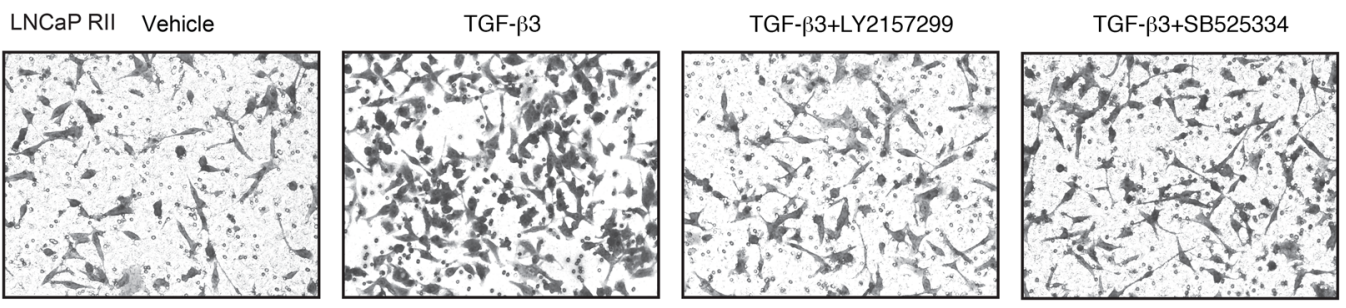

B

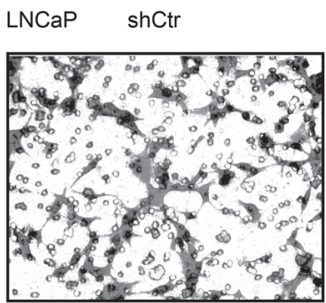

C

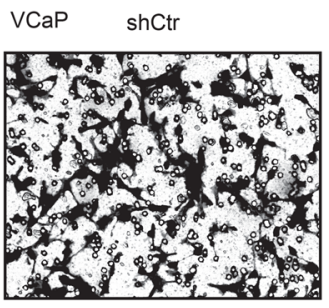

D
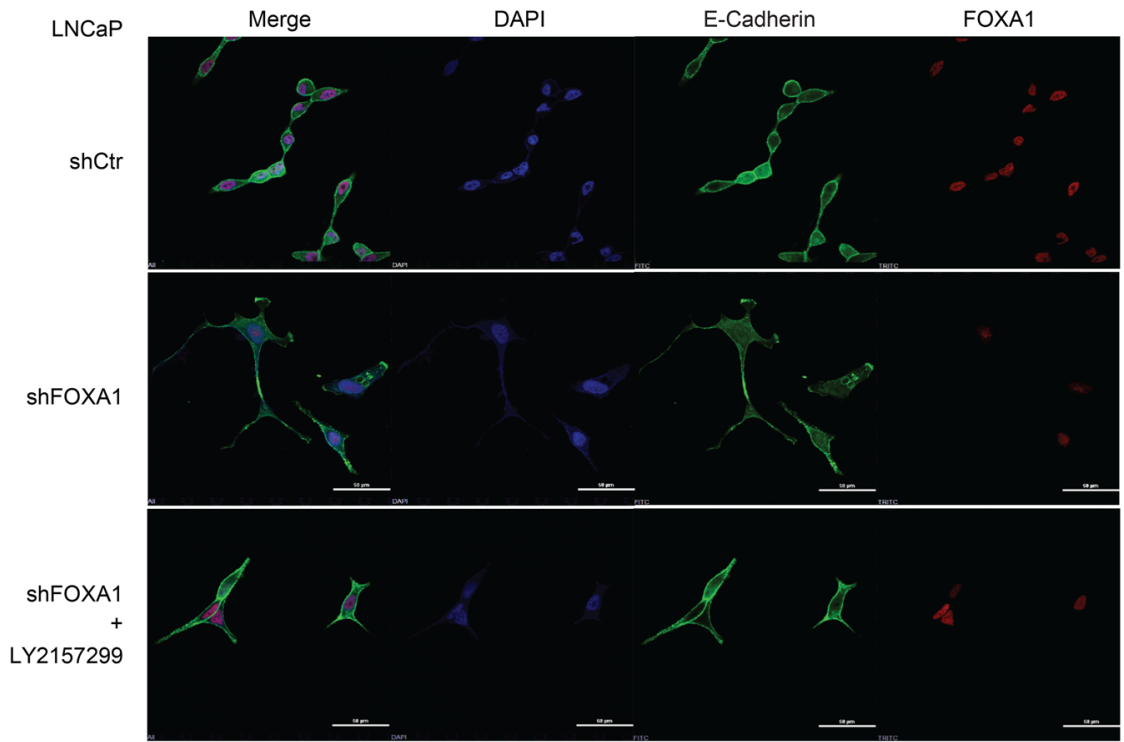

shFOXA1+LY2157299

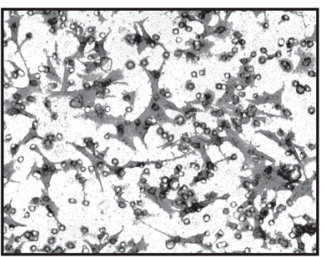

ShFOXA1
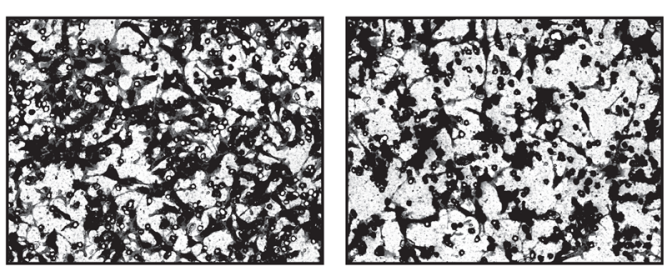

LCNaP
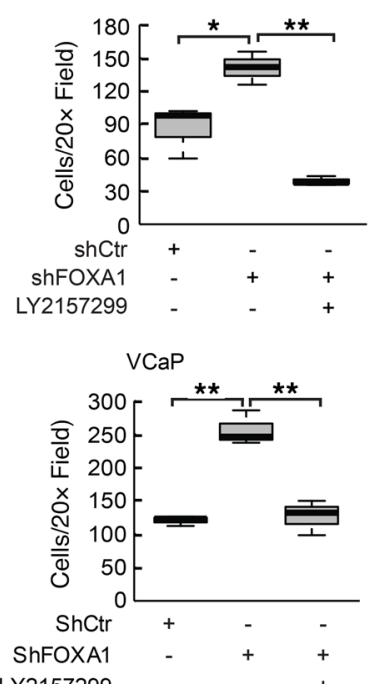

Figure 4. LY2157299 suppresses FOXA1-lossinduced cell invasion and EMT. (A)TCF- $\beta 3$ treatment enhances cell invasion, which can be blocked by LY2157299. LNCaP-RII cells were treated with $5 \mathrm{ng} / \mathrm{ml} \mathrm{TCF-} \beta 3$ ligand for 4 days, followed by $10 \mu \mathrm{M}$ LY2157299 treatment for 1 day and subjected to Matrigel invasion assay. (B and $\mathbf{C}$ ) Matrigel invasion assay of shCtr or shFOXA1 LNCaP (B) or VCaP (C) cells with or without $10 \mu \mathrm{M}$ LY2157299 treatment for 1 day. The number of invaded cells per $\times 20$ objective field was counted from 3 fields per conditions (right panels) $\left(n=3 ;{ }^{*} P<0.05\right.$; ${ }^{*} P<0.01$, 1-way ANOVA with Tukey's post hoc test). (D) Immunofluorescence staining showing EMT-like changes of cell morphology upon FOXA1 knockdown in LNCaP cells, which was reversed by LY2157299 treatment. Cells were stained for DAPI, FOXA1, and epithelial marker E-cadherin. Nuclei were stained with DAPI. Scale bars: $50 \mu \mathrm{m}$.

models. VCaP cells were inoculated subcutaneously into the right flanks of immune-deficient mice. Xenograft tumors were established in a majority of the mice after 4 weeks of inoculation, and the mice were castrated (Supplemental Figure 6A). Once CRPC tumors were stabilized, usually within 2 weeks, mice were randomized to receive treatment with vehicle, Enz alone, or Enz in combination with LY2157299. Measurements of tumor sizes over time revealed that Enz alone only slightly delayed the growth of xenograft CRPC tumors. On the other hand, xenograft tumors treated with LY2157299 and Enz drug combination failed to grow over the treatment window of 31 days (Figure 7A).

As previous studies have reported that Enz as a single agent induces xenograft tumor metastasis (29), and our data showed that TGF- $\beta 3$ signaling was inadvertently increased by Enz in cell lines, we examined tumor metastasis from the quantification of human alu sequences by real-time PCR (Alu-qPCR). Our results 
showed substantially increased rates of metastasis to femur in Enz-treated mice as compared with controls, whereas concurrent treatment with LY2157299 rescued this unfavorable metastatic effect (Figure 7B and Supplemental Figure 6B). Through IHC staining of xenograft tumors collected at the end of the study, we confirmed that Enz-treated mice indeed have induced pSMAD2, consistent with our in vitro data. This increase in PSMAD was fully abolished by concurrent LY2157299 treatment (Figure 7C and Supplemental Figure 6C). Further, cell proliferation was examined by Ki-67 staining, which showed relatively lower growth in mice treated with Enz and LY2157299, in agreement with the observed tumor growth rates (Figure 7D and Supplemental Figure 6D). In summary, these data demonstrated that inhibition of TGF- $\beta$ signaling using LY2157299 enhanced the efficacy of Enz in suppressing CRPC xenograft tumor growth and metastasis.

\section{Discussion}

FOXA1 is a pioneer factor that opens compact chromatin to facilitate the binding of other transcription factors such as AR and estrogen receptor $(24,30)$. We and others have shown that FOXA1 is required for the activation of prostate-specific gene expression $(31,32)$, and it plays dual roles in defining the AR transcriptional program (10-12). An increasing number of studies have reported that AR can bind to promoters or enhancers to directly suppress gene transcription $(25,26)$. In the present study, we integrated FOXA1-regulated gene expression with genomewide FOXA1 occupancy and found that FOXA1 can also act as a transcriptional repressor as well as a transcriptional activator. We identified TGFB3 as a gene that is strongly repressed by FOXA1. Using ChIP-qPCR and CRISPR/Cas9-mediated deletion, we further characterized a FOXA1-bound enhancer element that mediates this repressive effect. We showed that FOXA1-knockdown cells can secrete TGF- $\beta$ into the medium, which can activate the TGF- $\beta$ pathway in other PC cells through paracrine signaling. In addition to TGFB3, FOXA1 was also found to repress many genes of the TGF- $\beta$ signaling pathways, including the rate-limiting TGF$B R 2$. Consequently, we observed autocrine signaling leading to TGF- $\beta$ activation in FOXA1-knockdown LNCaP cells, although LNCaP normally has a low sensitivity to TGF- $\beta$ due to the lack of endogenous TGFBR2 expression. Taken together with our previous analysis of FOXA1-regulated gene expression $(13,14)$, FOXA1 appears to activate gene expression involved in cell cycle and growth, while it represses genes involved in EMT, cell motility, and immune response.

The expression of FOXA1 during PC progression reflects its dual roles in inducing androgen-dependent cell growth but inhibiting EMT and cell motility. At the mRNA level, multiple groups, including ours, have shown an initial increase of FOXA1 from benign to primary PC, but a decrease of FOXA1 once the disease progresses into CRPC status (10-12). In concordance with this finding, TMA analysis of primary PC tissues derived from radical prostatectomy has associated elevated levels of FOXA1 with enhanced AR binding and shorter time to biochemical recurrence (33). Similarly, another study reported that high FOXA1 expression significantly correlated with AR and tumor size, which may account for the observed higher rate of nodal metastasis for FOXA1-high tumors (34). It is important to note that patients in this cohort were treated primarily by surgery and none of them had received prior systemic therapy, thus representing primary PC with growth as its main feature. To truly examine FOXA1 expression in CRPC after ADT, we performed IHC staining of TMAs of metastatic CRPC from the warm autopsy program at the University of Washington and of primary PCs generated at Northwestern University. Our results revealed that a majority of primary PCs showed moderate to strong staining of FOXA1, consistent with RNA-seq profiling of tumor samples. By contrast, only half of metastatic CRPC tumors stained positive for FOXA1. Although we also observed more than $20 \%$ of metastatic CRPC with moderate to strong FOXA1 staining, a majority of CRPC has low or no FOXA1 expression. A previous study reported increased FOXA1 staining in CRPC (35). However, at least 23 of the 50 cases of CRPC in this study were obtained through transurethral resection of the prostate, and thus were not representative of metastatic diseases. In contrast, our study examined a much larger set of metastatic CRPC samples that were collected from distant organs through the warm autopsy program. Additional reasons for the discrepancy could include treatment histories, antibody used, staining conditions, and patient heterogeneities.

It has become evident in recent years that the TGF- $\beta$ pathway plays major roles in promoting EMT, prostate cancer cell motility, and tumor metastasis $(36,37)$. Overproduction of TGF- $\beta$ ligands or the TGF- $\beta$ target gene vimentin is correlated with poor clinical outcome in PC $(18,38)$. Recently, aberrant TGF- $\beta$ signaling was shown to drive CRPC in a mouse model of PC (39). Our study provides a mechanism to TGF- $\beta$ pathway activation in PC through FOXA1 downregulation. Our analysis of the TCGA and SU2C data sets confirmed elevated TGF- $\beta$ signaling in FOXA1-low tumors. However, TGF- $\beta$ signaling in PC tumors with reported FOXA1 mutations $(8,9)$ was not clearly inhibited but appeared also to be dependent on the level of FOXA1 expression, potentially due to heterogeneity of the mutations. Interestingly, Cai et al. recently identified genomic regions that interact with an enhancer at 8q24, a susceptibility loci for PC $(40,41)$. Interestingly, they showed that FOXA1 tends to occupy these 8q24-interacting genomic sites, which are involved in positive regulation of mesenchymal cell proliferation and the TGF- $\beta$ pathway (41), thereby suggesting FOXA1 regulation of the TGF- $\beta$ pathway.

There is a great interest in targeting the TGF- $\beta$ pathway, and the TGFBR1 inhibitor LY2157299 monohydrate has been under active preclinical and clinical development $(21,23)$. LY2157299 has also been shown to work well in combination with other drugs in liver cancer (42). In the present study, we demonstrate that LY2157299, when used in combination with Enz, has synergistic effects in suppressing PC cell growth and invasion in vitro and xenograft tumor growth and metastasis in immune-deficient mice. These results are further supported by a recent study from an independent group (43), which examined LY2157299 and Enz combination in immune-competent DNTGF $\beta$ RII TRAMP mice and observed significant suppression of prostate tumor growth through EMT reversion and redifferentiation. This is consistent with our observation of E-cadherin reexpression to the membrane accompanied by epithelial morphology. Altogether, our results provide the preclinical data and a strong rationale for clinical trials of Enz and LY2157299 for the treatment of patients with metastatic CRPC. 
A

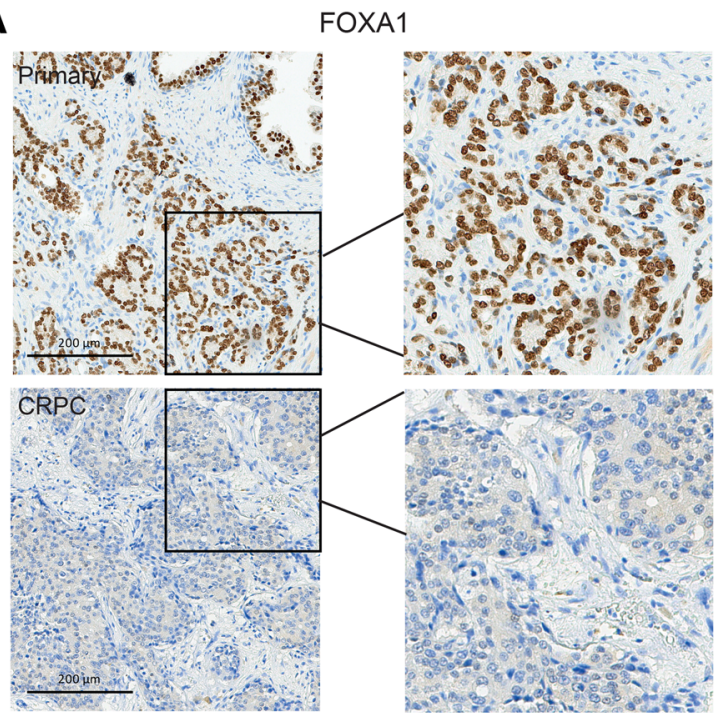

C

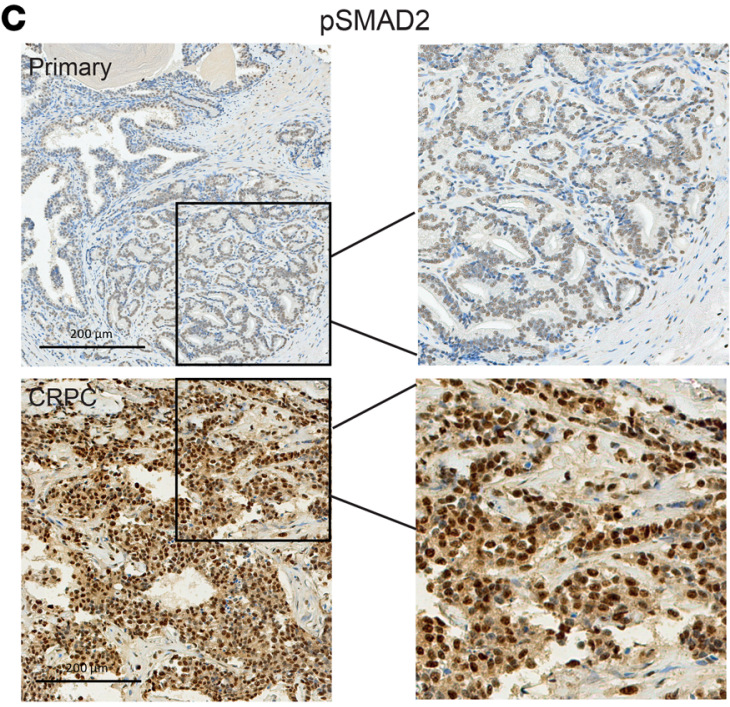

B

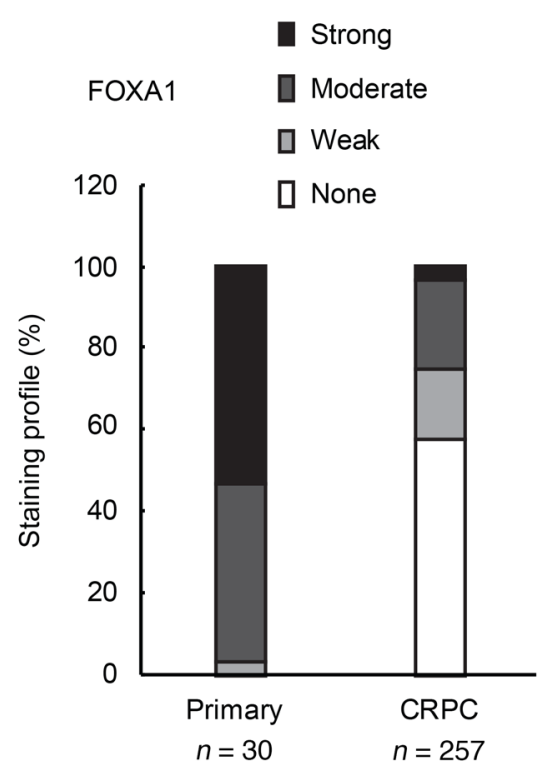

D

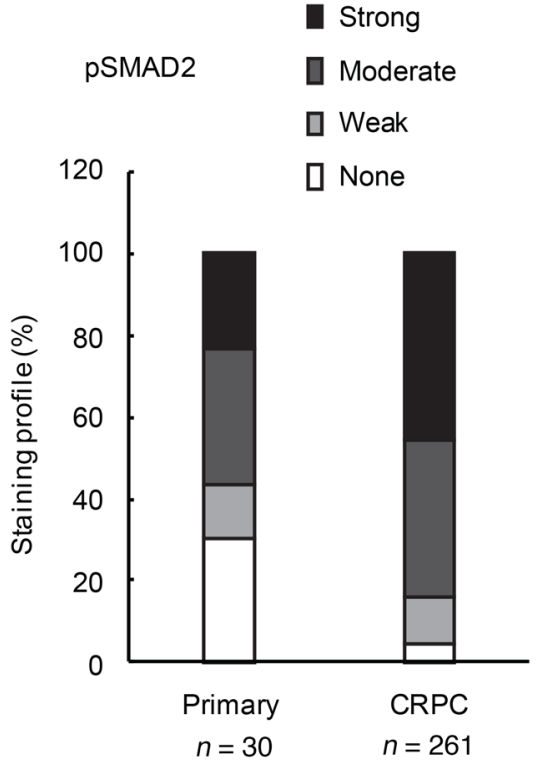

Figure 5. FOXA1 protein levels are decreased, whereas PSMAD is increased in metastatic CRPC as compared with primary PC. Immunohistochemistry staining was performed in TMA of primary prostate tumors and metastatic CRPC with indicated antibodies. (A and C) Representative images of FOXA1 (A) and pSMAD2 (C) staining in a primary tumor (top) and a CRPC tumor (bottom). Scale bars: $200 \mu \mathrm{m}$. (B and D) Quantification of FOXA1 (B) and pSMAD2 (D) staining intensities in primary PC and CRPC samples.

\section{Methods}

Tissue acquisition and tissue microarray analysis. Tissue microarrays containing metastatic CRPC specimens were obtained as part of the University of Washington Medical Center Prostate Cancer Donor Program, which is approved by the University of Washington Institutional Review Board. All specimens for IHC were formalin fixed (decalcified in formic acid for bone specimens), paraffin embedded, and examined histologically for the presence of nonnecrotic tumor. TMAs were constructed with 1-mm diameter duplicate cores $(n=538)$ from CRPC patient tissues $(n=92$ patients) consisting of visceral metastases and bone metastases $(n=269$ sites) from patients within 8 hours of death. TMAs of primary PCs
( $n=30$ patients, $n=30$ sites) were generated at the Northwestern University Pathology Core through the prostate SPORE program, and approved by the Northwestern University Institutional Review Board. Human TMA IHC staining was conducted using the Dako Autostainer Link 48 with enzyme-labeled biotin streptavidin system and the SIGMAFAST DAB Map Kit (MilliporeSigma). Antibodies used in IHC include anti-FoxA1 (1:400, ab23738, Abcam) and anti-pSMAD2 (1:2000, AB3849, Chemicon). Images were captured with TissueFax Plus from TissueGnostics, exported to TissueFAX viewer, and analyzed using Photoshop CS4 (Adobe). Immunostaining was quantified using a score of 0 to 3 for intensities of negative, weak, moderate, and strong. 
A

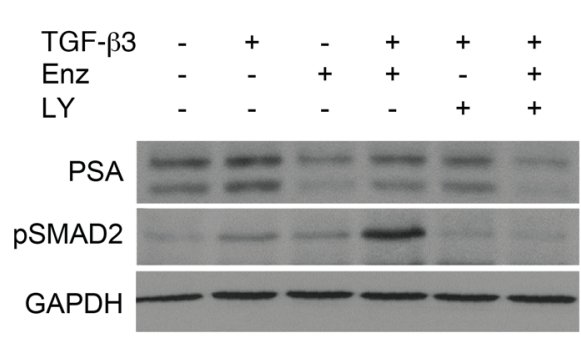

B

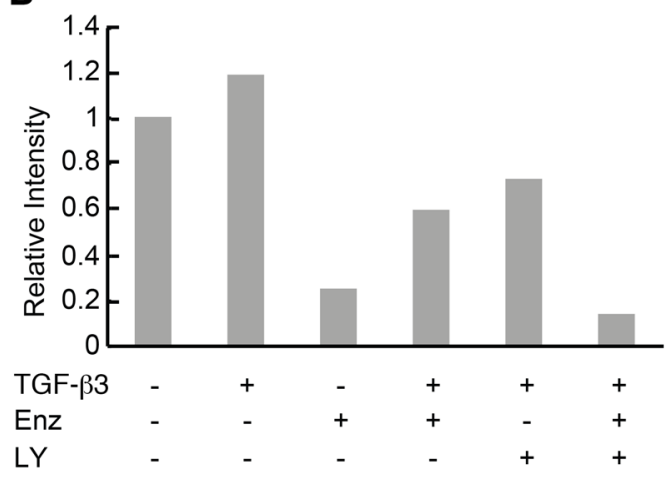

C
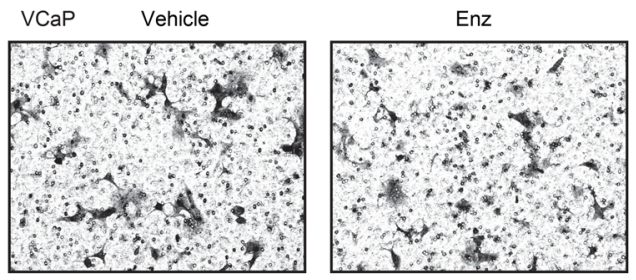

D
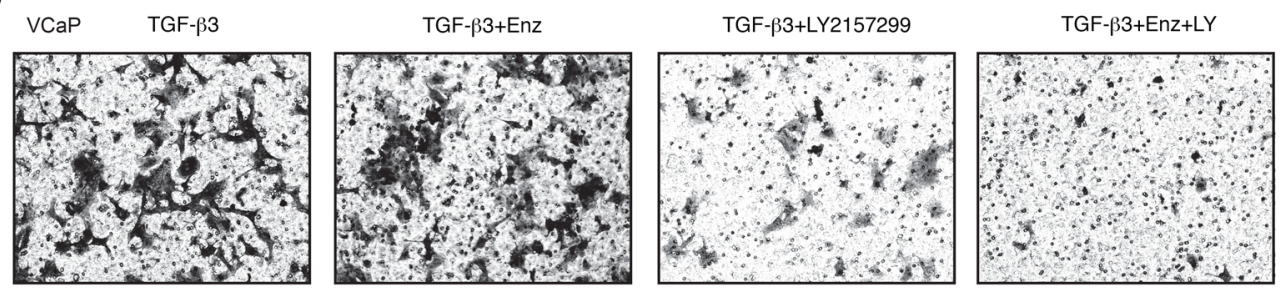

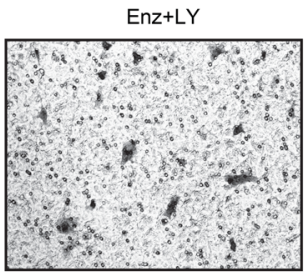

Figure 6. Enzalutamide and LY2157299 drug combination synergistically inhibit prostate cancer cell invasion. (A) Western blot analysis of PSA and pSMAD2 in VCaP cells treated with $5 \mathrm{ng} /$ $\mathrm{ml} \mathrm{TGF-} \beta 3,10 \mu \mathrm{M}$ Enz, and/or 10 $\mu$ M LY2157299 for 24 hours. (B) The relative PSA band intensity was quantified and normalized to $\mathrm{CAPDH}$. (C and $\mathbf{D})$ Matrigel assays of $\mathrm{VCaP}$ cells that were treated with vehicle control, $10 \mu \mathrm{M} \mathrm{Enz,} 10$ $\mu M$ LY2157299, or their combination in the absence (C) or presence of $5 \mathrm{ng} / \mathrm{ml}$ TCF- $\beta 3$ ligand (D). (E) Matrigel assays of LNCaP-RII stable cells treated with vehicle control, $10 \mu \mathrm{M}$ Enz, $10 \mu \mathrm{M}$ LY2157299, or their combination. Original magnification, C-E: $\times 20$.

E
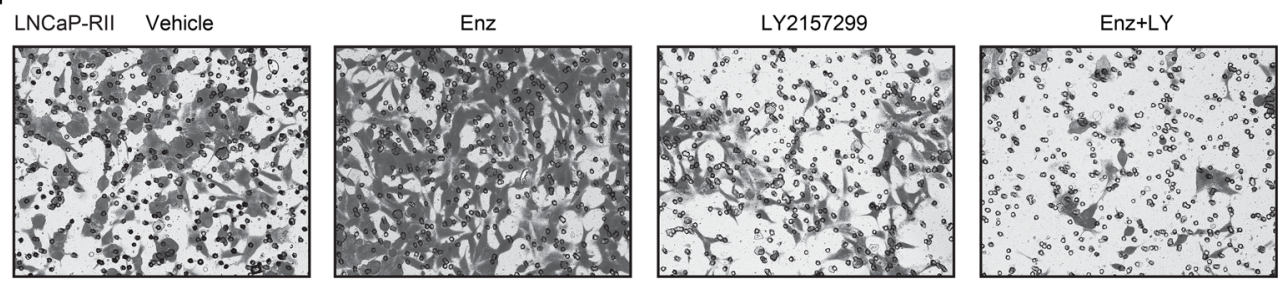

Cell culture and reagents. The PC cell lines $\mathrm{LNCaP}$ and VCaP were obtained from American Type Culture Collection (ATCC) and C4-2B cells were a gift from Qi Cao (Northwestern University, Chicago, Illinois). All cell lines were authenticated and tested free of mycoplasma. LNCaP and C4-2B cells were cultured in RPMI- 1640 with $10 \%$ fetal bovine serum (FBS) and 1\% penicillin/streptomycin. VCaP cells were cultured in DMEM with $10 \% \mathrm{FBS}$ and $1 \%$ penicillin/streptomycin. The cells were maintained at $37^{\circ} \mathrm{C}$ in a humidified incubator with $5 \% \mathrm{CO}_{2}$. Enz was purchased from Selleck Chemicals and dissolved in DMSO. LY2157299 was obtained from Selleck Chemicals and Eli Lilly and Company, and prepared according to the manufacturers' instructions. TGF- $\beta 3$ was purchased from R\&D Systems, and reconstituted at $20 \mu \mathrm{g} / \mathrm{ml}$ in $4 \mathrm{mM}$ sterile hydrochloric acid containing $1 \mathrm{mg} / \mathrm{ml}$ bovine serum albumin.

Conditioned media. LNCaP or VCaP FOXA1 stable knockdown and control cells were made by infecting control shRNA or FOXA1 shRNA lentivirus followed by 1 week of puromycin selection. Conditioned media were incubated 1 week prior to collection. Collected media were spun down to remove dead cells and heat-activated at $100^{\circ} \mathrm{C}$ to fully activate TGF- $\beta$ ligands, which upon secretion remain attached to the latency-associated propeptides through a noncovalent interaction.

Matrigel invasion assay. Matrigel was thawed on ice overnight in the cold room. Diluted Matrigel $(50 \mu \mathrm{l})$ was pipetted into the upper chamber of Transwell cell inserts ( $0.8 \mu \mathrm{m}$ pore size; Corning). The Matrigel was incubated at $37^{\circ} \mathrm{C}, 5 \% \mathrm{CO}_{2}$ for at least 30 minutes prior to addition of cells to the chamber. The cell suspension containing 300,000 cells/ml (LNCaP) or 600,000 cells/ml (VCaP) in serum-free RPMI or DMEM medium was prepared, and $100 \mu \mathrm{l}$ of the cell suspension was transferred into the upper chamber. The lower chamber contained $500 \mu \mathrm{l}$ complete growth medium with $40 \%$ FBS. After incubation at $37^{\circ} \mathrm{C}, 5 \% \mathrm{CO}_{2}$ for 72 hours, noninvading cells as well as the Matrigel from the interior of the inserts were gently removed using a cotton-tipped swab. The inserts were fixed and stained for 15 minutes in 25\% methanol containing $0.5 \%$ Crystal Violet. The images of invaded cells were captured under a 
A

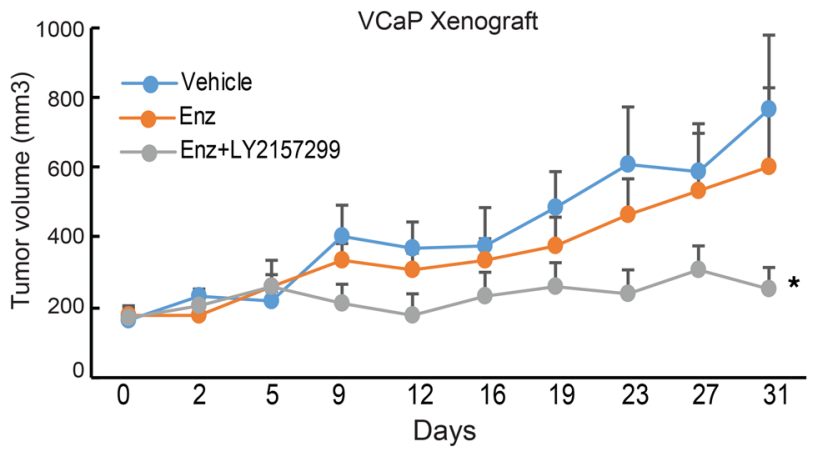

B

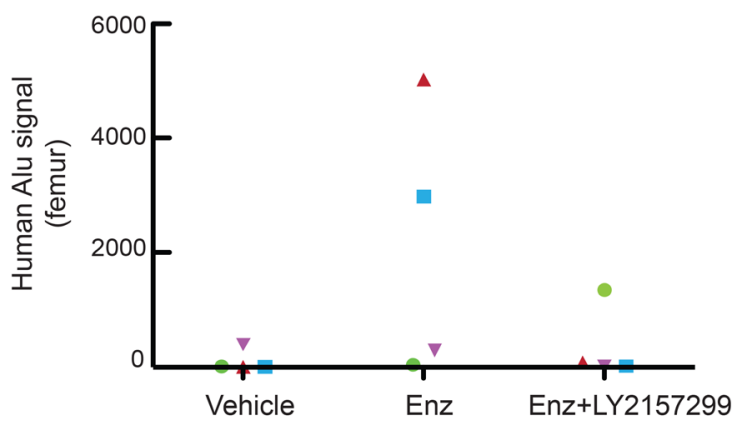

C

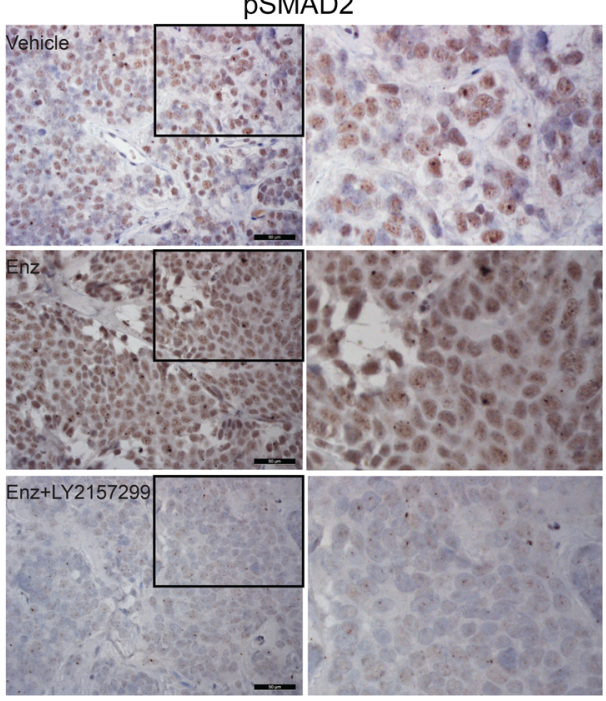

D

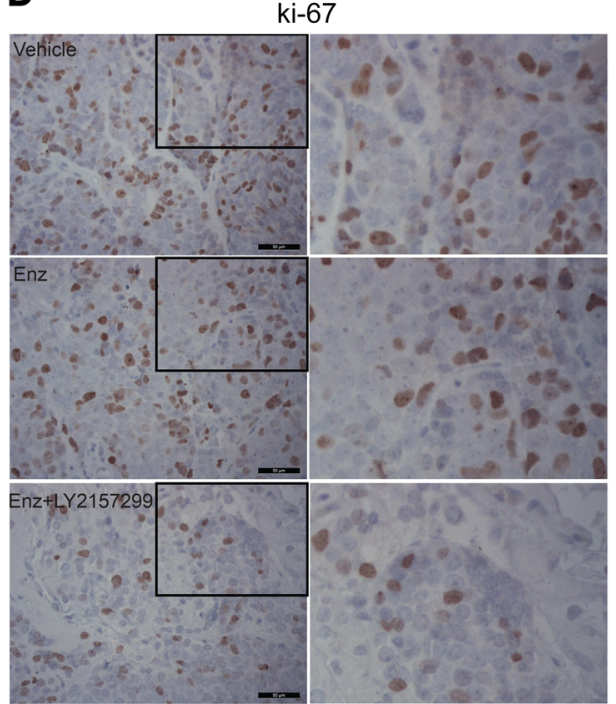

Figure 7. Enzalutamide and LY2157299 drug combination blocks xenograft prostate tumor growth and metastasis. (A) Castrated mice bearing VCaP xenografts received vehicle, Enz (oral, $10 \mathrm{mg} / \mathrm{kg}$ ) alone or in combination with LY2157299 (oral, $75 \mathrm{mg} / \mathrm{kg}$ ) 5 days per week for 33 days. Mean tumor volume $\pm \mathrm{SEM}$ is shown. Significance was calculated using ANOVA, $P<0.05$. (B) At the endpoint, mice were euthanized and femurs (bone marrow) were dissected. Genomic DNA were isolated and analyzed for metastasized cells by measuring human Alu sequence (by Alu-qPCR). (C and D) Immunohistochemistry was performed in tumor sections isolated from xenograft mice with the indicated antibodies and $\mathrm{H} \& \mathrm{E}$ staining. Representative images are shown. Scale bars: $50 \mu \mathrm{m}$. brightfield microscope, and the number of invaded cells per field view was counted using the cell counter plugins in Image J (NIH).

Gene expression array analysis. Total RNAs were isolated using TRIzol reagent (Invitrogen). The integrity of the RNA was monitored using the Bioanalyzer 2100 (Agilent). Microarray profiling was conducted using the HumanHT-12 v 4.0 Expression BeadChip (Illumina). Bead-level data were preprocessed using GenomeStudio (Illumina), and the expression values were quantile-normalized using the bead array package from Bioconductor. Differentially expressed genes were identified using a 2-fold cutoff.

ChIP-seq, RNA-seq, and analysis. Previously published FOXA1 ChIP-seq data (NCBI Gene Expression Omnibus database, GSE55007) were reanalyzed. For RNA-seq, total RNA was isolated from cells using the PureLink RNA Mini Kit (Thermo Fisher Scientific). RNA-seq libraries were prepared from $0.5 \mu \mathrm{g}$ high-quality DNA-free total RNA by using the NEBNext Ultra RNA Library Prep Kit (New England Biolabs), according to the manufacturer's instructions. The libraries were sequenced using the Illumina Hi-Seq platform. RNA-seq reads were mapped to the NCBI human genome GRCh38 using STAR version 1.5.2 (44). Raw counts of genes were calculated using STAR. RPKM values were calculated using an in-house Perl script. Differential gene expression was analyzed using the R Bioconductor DESeq2 package (45), which uses shrinkage estimation for dispersions and fold changes to improve stability and interpretability of estimates.
All high-throughput data, including microarray and RNA-seq, have been deposited to NCBI's Gene Expression Omnibus database (GEO GSE119759).

CRISPR/Cas9-mediated deletion of enhancers. The sgRNAs were designed using the MIT CRISPR design software (http://crispr.mit. edu). The sgRNA oligos were synthesized by Integrated DNA Technologies (IDT) and cloned into the lentiviral transfer plasmid LentiCRISPRv2 (a gift from Jon A. Oyer, Northwestern University, Chicago, Illinois). Lentiviral particles were produced in 293T cells and collected to infect LNCaP cells. Puromycin was added 24 hours after infection and selected for another 48 hours. Cells were then subjected to control or FOXA1 knockdown and another 2 days of puromycin selection. Total genomic DNA was then extracted using the QIAamp DNA Mini Kit (Qiagen), and RNA was isolated using Trizol (Invitrogen). PCR of genomic DNA was performed using the indicated primers flanking the sgRNA target sites. PCR products of the WT and deleted alleles were examined and purified by agarose gel and sequence-validated by Sanger sequencing method. RNA was subjected to qRT-PCR analysis of TGFB3 gene expression.

Murine prostate tumor xenograft model. Mouse handling and experimental procedures were approved by the Center for Animal and Comparative Medicine at the Northwestern University School of Medicine in accordance with the NIH Guide for the Care and Use of Laboratory Animals (National Academies Press, 2011) and the Animal Welfare Act. 
SCID.CB17 male mice at 3-4 weeks old were purchased from Charles River Laboratories. Briefly, a suspension of $\mathrm{VCaP}$ cells ( 5 million cells in PBS 1:1 mixed with Matrigel) was inoculated into the right flank of the mice. Four weeks later, mice bearing tumors of approximately 150 $\mathrm{mm}^{3}$ were surgically castrated. Approximately 2 weeks later, regressed tumors grew back. Mice were then randomized and treated with vehicle, enzalutamide (Enz; $10 \mathrm{mg} / \mathrm{kg}$ ), or a combination with LY2157299 (75 mg/kg). Enz was administered daily by oral gavage. LY2157299 was administered twice daily by oral gavage. Tumor volumes were measured every 3 days with digital calipers, using the formula: $\mathrm{V}=\mathrm{L} \times$ $\mathrm{W} 2 / 2$ (V, $\mathrm{mm}^{3}$; L, mm; W, mm).

Statistics. Two-tailed paired $t$ tests were used to assess statistical significances in quantitative RT-PCR experiments and cell functional assays. One-way ANOVA was used to determine statistically significant differences across treatment groups in the xenograft study. $P$ less than 0.05 indicates statistical significance. The statistical significance of cell invasion was assessed by 1-way ANOVA with Tukey's post hoc test.

Study approval. The Northwestern University IACUC (Chicago, IL) approved all animal studies. The metastatic CRPC TMA was approved and provided by the University of Washington Medical Center through the Prostate Cancer Donor Rapid Autopsy Program.

\section{Author contributions}

JY and BS conceived the project and designed the experiment. BS, SHP, KF, SL, YL, YAY, SS, and XL performed the experiments. JCZ and JY conducted bioinformatics and statistics analysis. RLV, $\mathrm{CM}, \mathrm{WC}$, and $\mathrm{XY}$ assisted with tissue microarray acquisition and experiment. SAA consulted on the animal experiment. WC and TMK provided critical discussions of the project. JY, BS, and SHP generated the figures and wrote the manuscript.

\section{Acknowledgments}

We thank Robin Leikin for helpful discussions of the project, Jonathan Anker and Young A. Yoo for help with the mouse work, and
Bert Vogelstein for providing SBE-luc plasmid. The compound LY2157299 monohydrate was provided by Eli Lilly and Company. This work was supported in part by NIH grant R01CA172384 (to JY), NIH grant R50CA211271 (to JCZ), NIH prostate SPORE grant P50CA180995 (to JY), and Prostate Cancer Foundation grant 2017CHAL2008 (to JY, JCZ, YL, and CM). Imaging work was performed at the Northwestern University Center for Advanced Microscopy and IHC staining was performed at the Pathology Core Facility, and generously supported by National Cancer Institute grant P30CA060553 awarded to the Robert H. Lurie Comprehensive Cancer Center. The metastatic CRPC TMA was provided by the University of Washington Medical Center Prostate Cancer Donor Rapid Autopsy Program. We thank the patients and their families, Celestia Higano, Evan Yu, Pete Nelson, Elahe Mostaghel, Heather Cheng, Bruce Montgomery, Mike Schweizer, Paul Lange, Funda Vakar-Lopez, Xiaotun Zhang, Martine Roudier, Lawrence True, and the rapid autopsy teams for their contributions. The rapid autopsy program was supported by the Department of Defense Prostate Cancer Research Program Award (W81XWH-14-2-0183), the Pacific Northwest Prostate Cancer SPORE (P50CA97186), the PO1 NIH grant (PO1CA163227), and the Institute for Prostate Cancer Research.

Address correspondence to: Jindan Yu, Division of Hematology/ Oncology, Department of Medicine, Robert H. Lurie Comprehensive Cancer Center, Northwestern University, Feinberg School of Medicine, 303 E. Superior Street, Lurie 5-117, Chicago, Illinois 60611, USA. Phone: 312.503.1761; Email:jindan-yu@northwestern.edu.

BS's present address is: School of Life Sciences, Zhengzhou University, Zhengzhou, China.

TMK's present address is: Department of Medicine, Hematology/ Oncology, Rush University, Chicago, Illinois, USA.
1. Siegel RL, Miller KD, Jemal A. Cancer statistics, 2018. CA Cancer J Clin. 2018;68(1):7-30.

2. de Bono JS, et al. Abiraterone and increased survival in metastatic prostate cancer. $N$ Engl J Med. 2011;364(21):1995-2005.

3. Scher HI, et al. Increased survival with enzalutamide in prostate cancer after chemotherapy. N Engl JMed. 2012;367(13):1187-1197.

4. $\mathrm{Mu} \mathrm{P}$, et al. SOX2 promotes lineage plasticity and antiandrogen resistance in TP53- and RB1-deficient prostate cancer. Science. 2017;355(6320):84-88.

5. Ku SY, et al. Rb1 and Trp53 cooperate to suppress prostate cancer lineage plasticity, metastasis, and antiandrogen resistance. Science. 2017;355(6320):78-83.

6. Chen CD, et al. Molecular determinants of resistance to antiandrogen therapy. Nat Med. 2004;10(1):33-39.

7. Yuan X, Balk SP. Mechanisms mediating androgen receptor reactivation after castration. Urol Oncol. 2009;27(1):36-41.

8. Grasso CS, et al. The mutational landscape of lethal castration-resistant prostate cancer. Nature. 2012;487(7406):239-243.

9. Cancer Genome Atlas Research Network.
The molecular taxonomy of primary prostate cancer. Cell. 2015;163(4):1011-1025.

10. Wang D, et al. Reprogramming transcription by distinct classes of enhancers functionally defined by eRNA. Nature. 2011;474(7351):390-394.

11. Sahu B, et al. Dual role of FoxA1 in androgen receptor binding to chromatin, androgen signalling and prostate cancer. EMBO J. 2011;30(19):3962-3976.

12. Jin HJ, Zhao JC, Wu L, Kim J, Yu J. Cooperativity and equilibrium with FOXA1 define the androgen receptor transcriptional program. Nat Commun. 2014;5:3972.

13. Jin HJ, Zhao JC, Ogden I, Bergan RC, Yu J. Androgen receptor-independent function of FoxA1 in prostate cancer metastasis. Cancer Res. 2013;73(12):3725-3736.

14. Kim J, et al. FOXA1 inhibits prostate cancer neuroendocrine differentiation. Oncogene. 2017;36(28):4072-4080.

15. Jones E, Pu H, Kyprianou N. Targeting TGF-beta in prostate cancer: therapeutic possibilities during tumor progression. Expert Opin Ther Targets. 2009;13(2):227-234

16. Massagué J. TGFbeta in cancer. Cell. 2008;134(2):215-230.
17. Padua D, Massagué J. Roles of TGFbeta in metastasis. Cell Res. 2009;19(1):89-102.

18. Zhang Q, et al. Nuclear factor-kappaB-mediated transforming growth factor-beta-induced expression of vimentin is an independent predictor of biochemical recurrence after radical prostatectomy. Clin Cancer Res. 2009;15(10):3557-3567.

19. Ye H, et al. Human bone marrow-derived mesenchymal stem cells produced TGFbeta contributes to progression and metastasis of prostate cancer. Cancer Invest. 2012;30(7):513-518.

20. Walker L, Millena AC, Strong N, Khan SA. Expression of TGF $\beta 3$ and its effects on migratory and invasive behavior of prostate cancer cells: involvement of PI3-kinase/AKT signaling pathway. Clin Exp Metastasis. 2013;30(1):13-23.

21. Yingling JM, et al. Preclinical assessment of galunisertib (LY2157299 monohydrate), a first-inclass transforming growth factor- $\beta$ receptor type I inhibitor. Oncotarget. 2018;9(6):6659-6677.

22 . Kovacs RJ, et al. Cardiac safety of TGF- $\beta$ receptor I kinase inhibitor LY2157299 monohydrate in cancer patients in a first-in-human dose study. Cardiovasc Toxicol. 2015;15(4):309-323.

23. Rodon J, et al. First-in-human dose study of the novel transforming growth factor- $\beta$ receptor 
I kinase inhibitor LY2157299 monohydrate in patients with advanced cancer and glioma. Clin Cancer Res. 2015;21(3):553-560.

24. Lupien M, et al. FoxA1 translates epigenetic signatures into enhancer-driven lineage-specific transcription. Cell. 2008;132(6):958-970.

25. Zhao JC, et al. Cooperation between polycomb and androgen receptor during oncogenic transformation. Genome Res. 2012;22(2):322-331.

26. Cai C, et al. Androgen receptor gene expression in prostate cancer is directly suppressed by the androgen receptor through recruitment of lysine-specific demethylase 1. Cancer Cell. 2011;20(4):457-471.

27. Yamashita S, et al. Methylation silencing of transforming growth factor-beta receptor type II in rat prostate cancers. Cancer Res. 2008;68(7):2112-2121.

28. Chipuk JE, et al. The androgen receptor represses transforming growth factor-beta signaling through interaction with Smad3. J Biol Chem. 2002;277(2):1240-1248.

29. Asangani IA, et al. Therapeutic targeting of BET bromodomain proteins in castration-resistant prostate cancer. Nature. 2014;510(7504):278-282.

30. Jozwik KM, Carroll JS. Pioneer factors in hormone-dependent cancers. Nat Rev Cancer. 2012;12(6):381-385.

31. Gao N, et al. Forkhead box A1 regulates prostate ductal morphogenesis and promotes epithelial cell maturation. Development. 2005;132(15):3431-3443.

32. Wang $\mathrm{Q}$, et al. Androgen receptor regulates a distinct transcription program in androgen-independent prostate cancer. Cell. 2009;138(2):245-256.

33. Robinson JL, et al. Elevated levels of FOXA1 facilitate androgen receptor chromatin binding resulting in a CRPC-like phenotype. Oncogene. 2014;33(50):5666-5674.

34. Jain RK, Mehta RJ, Nakshatri H, Idrees MT, Badve SS. High-level expression of forkhead-box protein $\mathrm{A} 1$ in metastatic prostate cancer. Histopathology. 2011;58(5):766-772.

35 . Gerhardt J, et al. FOXA1 promotes tumor progression in prostate cancer and represents a novel hallmark of castration-resistant prostate cancer. Am J Pathol. 2012;180(2):848-861.

36. Ao M, Williams K, Bhowmick NA, Hayward SW. Transforming growth factor-beta promotes invasion in tumorigenic but not in nontumorigenic human prostatic epithelial cells. Cancer Res. 2006;66(16):8007-8016.

37. Shiota M, et al. Clusterin mediates TGF- $\beta$ induced epithelial-mesenchymal transition and metastasis via Twist 1 in prostate cancer cells. Cancer Res. 2012;72(20):5261-5272.

38. Wikström P, Stattin P, Franck-Lissbrant I, Damber JE, Bergh A. Transforming growth factor beta1 is associated with angiogenesis, metastasis, and poor clinical outcome in prostate cancer.
Prostate. 1998;37(1):19-29.

39. Pu H, Begemann DE, Kyprianou N. Aberrant TGF- $\beta$ signaling drives castration-resistant prostate cancer in a male mouse model of prostate tumorigenesis. Endocrinology. 2017;158(6):1612-1622.

40. Amundadottir LT, et al. A common variant associated with prostate cancer in European and African populations. Nat Genet. 2006;38(6):652-658.

41. Cai M, Kim S, Wang K, Farnham PJ, Coetzee GA, $\mathrm{Lu} \mathrm{W.} \mathrm{4C-seq} \mathrm{revealed} \mathrm{long-range} \mathrm{interactions} \mathrm{of}$ a functional enhancer at the $8 \mathrm{q} 24$ prostate cancer risk locus. Sci Rep. 2016;6:22462.

42. Serova M, et al. Effects of TGF-beta signalling inhibition with galunisertib (LY2157299) in hepatocellular carcinoma models and in ex vivo whole tumor tissue samples from patients. Oncotarget. 2015;6(25):21614-21627.

43. Paller C, Pu H, Begemann DE, Wade CA, Hensley PJ, Kyprianou N. TGF-beta receptor I inhibitor enhances response to enzalutamide in a preclinical model of advanced prostate cancer [published online ahead of print August 28th, 2018]. Prostate. https://doi.org/10.1002/pros.23708.

44. Dobin A, et al. STAR: ultrafast universal RNA-seq aligner. Bioinformatics. 2013;29(1):15-21.

45. Love MI, Huber W, Anders S. Moderated estimation of fold change and dispersion for RNA-seq data with DESeq2. Genome Biol. 2014;15(12):550. 